\title{
CARACTERIZAÇÃO DE INTERNAÇÕES DE DEPENDENTES QUÍMICOS EM UMA UNIDADE DE REABILITAÇÃO
}

\author{
Aline Cristina Zerwes Ferreira ${ }^{1}$, Fernanda Carolina Capistrano ${ }^{2}$, Mariluci Alves Maftum³, \\ Luciana Puchalski Kalinke ${ }^{4}$, Ana Lúcia Cardoso Kirchhof ${ }^{5}$
}

\begin{abstract}
RESUMO: Pesquisa transversal e retrospectiva que utilizou a técnica de pesquisa documental, cujo objetivo foi caracterizar as internações de dependentes químicos em uma unidade de reabilitação. Foram coletados dados de 350 prontuários de dependentes químicos, referentes ao ano de 2010. Quanto à caracterização dos sujeitos, a média etária foi de 35,8 anos, 39,4\% estavam solteiros, $67,3 \%$ cursaram até o ensino fundamental e $45,1 \%$ eram desempregados. O álcool motivou a busca por tratamento em $45,1 \%$ dos pacientes; 49,4\% foram diagnosticados pela dependência de múltiplas drogas. A internação para 97,1\% foi voluntária; 40,4\% dos responsáveis foram os pais; a alta clínica prevaleceu em 55,4\% e, após alta, 86\% foram encaminhados a serviços extra-hospitalares. Dentre os participantes, $71,7 \%$ tiveram internamentos anteriores e $29,9 \%$ realizaram outros tipos de tratamento. Os resultados demonstram que é imprescindível a manutenção do tratamento após a internação para a busca da abstinência permanente. PALAVRAS-CHAVE: Enfermagem; Saúde mental; Transtornos relacionados ao uso de substâncias.

\section{CHARACTERIZATION OF THE HOSPITALIZATION OF DRUG ADDICTS IN A REHABILITATION UNIT}

\begin{abstract}
This cross-sectional, retrospective research used the technique of documental research. Its objective was to characterize the hospitalization of drug addicts in a rehabilitation unit. Data was collected from 350 patient records belonging to drug addicts, referring to 2010. Regarding the characterization of the subjects, the average age was 35.8 years, 39.4\% were single, $67.3 \%$ had only studied at primary school level and $45.1 \%$ were unemployed. Alcohol was the motive for treatment in $45.1 \%$ of the patients; $49.4 \%$ were diagnosed as dependent on multiple drugs. Hospitalization was voluntary for $97.1 \%$; $40.4 \%$ of those responsible were the parents; clinical discharge predominated in $55.4 \%$ and, after discharge, $86 \%$ were referred to community services. Among the participants, $71.7 \%$ had been hospitalized before and $29.9 \%$ had undertaken other types of treatment. The results demonstrate that to aim for permanent abstinence, it is essential to maintain treatment after the hospitalization.
\end{abstract}

KEYWORDS: Nursing; Mental health; Disorders related to substance use.

\section{CARACTERIZACIÓN DE INTERNACIONES DE DEPENDIENTES QUÍMICOS EN UNA UNIDAD DE REHABILITACIÓN}

RESUMEN: Investigación transversal y retrospectiva que utilizó la técnica de investigación documental, cuyo objetivo fue caracterizar las internaciones de dependientes químicos en una unidad de rehabilitación. Fueron obtenidos datos de 350 prontuarios de dependientes químicos que se refieren al año de 2010. Cuanto a la caracterización de los sujetos, la media etaria fue de 35,8 años, 39,4\% estaban solteros, 67,3\% estudiaron hasta la enseñanza fundamental y 45,1\% estaban desempleados. El álcohol motivó la búsqueda por tratamiento en $45,1 \%$ de los pacientes; 49,4\% fueron diagnosticados por la dependencia de múltiplas drogas. La internación para 97,1\% fue voluntaria; 40,4\% de los responsables fueron los padres; el alta clínica prevaleció en 55,4\% y, después del alta, $86 \%$ fueron encaminados a servicios extrahospitalares. Entre los participantes, 71,7\% tuvieron internamientos anteriores y 29,9\% realizaron otros tipos de tratamiento. Los resultados demuestran que es imprescindible la manutención del tratamiento después de la internación para la búsqueda de la abstinencia permanente.

PALABRAS CLAVES: Enfermería; Salud mental; Trastornos relacionados al uso de sustancias.

\footnotetext{
${ }^{1}$ Acadêmica de Enfermagem da Universidade Federal do Paraná - UFPR. Membro do Núcleo de Estudos, Pesquisa e Extensão em Cuidado Humano em Enfermagem - NEPECHE. Bolsista CNPq de iniciação científica.

${ }^{2}$ Acadêmica de Enfermagem da UFPR. Membro do NEPECHE. Bolsista voluntária de Iniciação Científica.

${ }^{3}$ Enfermeira. Doutora em Enfermagem. Professora do Curso de Graduação em Enfermagem, Coordenadora e Professora do Programa de Pós-Graduação em Enfermagem - PPGENF UFPR. Vice-Coordenadora do NEPECHE.

${ }^{4}$ Enfermeira. Doutora em Ciências da Saúde. Professora do Curso de Graduação em Enfermagem, Vice-coordenadora e Professora do PPGENF UFPR. Membro do Grupo de Estudos Multiprofissionais em Saúde do Adulto - GEMSA.

${ }^{5}$ Enfermeira. Doutora em Enfermagem. Professora Visitante do PPGENF UFPR.
}

Autor correspondente:

Recebido: 28/04/2012

Mariluci Alves Maftum Aprovado: 19/07/2012

Universidade Federal do Paraná

Rua Lothário Meissner, 632 - 80210-170 -Curitiba-PR-Brasil

E-mail:maftum@ufpr.br

Cogitare Enferm. 2012 Jul/Set; 17(3):444-51 


\section{INTRODUÇÃO}

O uso indiscriminado de substâncias psicoativas assumiu proporções alarmantes nas últimas décadas caracterizando, atualmente, uma grave doença social epidêmica. Isso porque, o consumo de drogas altera o funcionamento do sistema nervoso central e gera deficits de percepção, humor e consciência. Por conseguinte, o indivíduo perde a capacidade de controle e subordinase a elas ${ }^{(1)}$. Conforme o Escritório das Nações Unidas contra Drogas e Crimes, aproximadamente 210 milhões de pessoas, correspondendo a $4,8 \%$ da população mundial, com idade entre 15 e 64 anos, consumiram ao menos uma vez substâncias ilícitas nos últimos anos ${ }^{(2)}$. Entrementes, a Organização Mundial da Saúde (OMS) estima que 15,3 milhões de pessoas, no mundo, possuem transtornos mentais decorrentes desse uso $^{(3)}$.

No Brasil esses dados se equiparam ${ }^{(4)}$, e visando lidar com tal situação, foi desenvolvida a Política de Atenção Integral a usuários de álcool e outras drogas. Esta Política tem o foco na mobilização social para a prevenção e tratamento do consumo de drogas, na reabilitação e na reinserção social do usuário. A ênfase do tratamento está em serviços extra-hospitalares ${ }^{(4)}$. No que concerne a estes serviços, para o tratamento de dependentes químicos, a realidade brasileira demonstra baixa adesão dos usuários e insuficiência de estruturas para suprir a demanda que possibilite assistência de qualidade. Tais fatos dificultam a manutenção do usuário em tratamento no seu meio social e familiar, contribuindo para a internação(5).

Quando a internação se torna inevitável, inicialmente sua finalidade se volta para a desintoxicação do usuário. Entretanto, em todos os momentos do tratamento é função de todos os profissionais da equipe de saúde auxiliá-lo na manutenção da abstinência e na reabilitação. A intervenção a partir de tratamento estruturado, concomitante à retirada do indivíduo do convívio social por um curto período de tempo, faz com que o paciente repense a vida, e as áreas nela afetadas, sob uma nova perspectiva, uma vez que a conscientização do problema é fundamental para a continuidade do tratamento ${ }^{(6)}$. Deste modo, compreende-se que esse tipo de tratamento reporta a um importante desafio no que se refere à adesão. Estudos apontam índices elevados de abandono ao tratamento da dependência química, agravando ainda mais a possibilidade de recuperação desses indivíduos ${ }^{(7-8)}$.

A maior limitação da recuperação do dependente químico se deve à propensão à reincidência do consu- mo de drogas. Assim, após a internação, muitos deles, experimentam a recaída, como retorno do consumo das drogas na mesma proporção que a precedente ao internamento. Comumente, essa reincidência resulta na reinternação ${ }^{(9)}$.

Este estudo se justifica devido a importância da temática caracterizada atualmente no mundo como um problema de saúde e social, ao caráter crônico e de difícil recuperação do dependente químico. Assim, esta pesquisa teve como objetivo caracterizar as internações de dependentes químico em uma unidade de reabilitação.

\section{MÉTODO}

Pesquisa transversal, retrospectiva que utilizou a técnica de pesquisa documental, desenvolvida no período de dezembro de 2010 a fevereiro de 2011, em uma unidade de reabilitação para dependência química pertencente a um Hospital Psiquiátrico do Paraná. Esta unidade dispõe de 35 leitos e admite somente pacientes do sexo masculino, com idade mínima de 18 anos. Os leitos são classificados como de curta permanência (aproximadamente 45 dias) e são indicados como último recurso de tratamento, quando os tratamentos extra-hospitalares não se tornaram favoráveis ou suficientes. A amostra da pesquisa foi constituída por 350 prontuários; do total de 380 dependentes químicos admitidos de janeiro a dezembro de 2010, foram excluídos 30 por reinternação.

Os dados foram registrados em formulário estruturado, previamente testado e adaptado à realidade investigada com as seguintes variáveis: dados sociodemográficos, histórico de tratamentos de dependência química e internação atual. Os dados coletados foram codificados numericamente e armazenados em uma planilha de banco de dados do programa Microsoft Excel ${ }^{\circledR}$. Na análise quantitativa utilizou-se o pacote computacional Static ${ }^{\circledR}$ (versão 8.0). Os resultados foram descritos e analisados pelo método quantitativo descritivo e obtidos por meio de cálculos relativos, a partir do total válido de informações, uma vez que não havia todas as informações nos prontuários. Os resultados foram expostos por médias, valores mínimos e máximos, ou por frequências e percentuais. O teste exato de Fischer foi aplicado na avaliação da associação entre as variáveis qualitativas dicotômicas. Já para avaliar a correlação entre grupos relacionados a variáveis quantitativas, aplicou-se o modelo de análise de variância com um fator. Foi estabelecido o nível de significância mínimo de $\mathrm{p}<0,05$ (5\%). 
Esta pesquisa integra o projeto "O reiternamento de dependentes químicos em uma unidade de reabilitação: causas e motivações da recaída", aprovado no Comitê de Ética do Setor de Ciências da Saúde da Universidade Federal do Paraná, sob o registro CEP/SD: 904.029.10.03; CAAE: 0825.0.000.091-10. Os aspectos éticos foram salvaguardados e o início da coleta dos dados ocorreu após autorização formal da diretoria da instituição.

\section{RESULTADOS}

Considerando as características sociodemográficas dos dependentes químicos analisados a média de idade (n-350) foi de 35,8 anos (desvio-padrão: 11,9). No que se refere ao estado civil (n-345), houve o predomínio de solteiros em 39,4\%. Quanto ao nível de escolaridade (n-98), 67,3\% dos pacientes tem formação de ensino fundamental. Os dados relativos à ocupação (n-135), apontam que o desemprego é preeminente em $45,2 \%$. A tabela 1 destaca os dados da admissão na unidade de desintoxicação. Observa-se que o motivo da internação com maior prevalência é o uso crônico do álcool (n-158) $45,1 \%$, seguido pelo uso crônico de múltiplas drogas

Tabela 1 - Distribuição dos dados da admissão hospitalar de dependentes químicos. Curitiba, 2011

\begin{tabular}{lcc}
\hline Dados da admissão & N & \% \\
\hline Motivo da internação & & \\
Uso crônico de álcool & 158 & 45,1 \\
Uso crônico de múltiplas drogas & 129 & 36,9 \\
Uso crônico de crack & 58 & 16,6 \\
Outros & 5 & 1,4 \\
Total & 350 & $100 \%$ \\
Tipo de Internação & & \\
Voluntária & 340 & 97,1 \\
Involuntária & 10 & 2,9 \\
Total & 350 & $100 \%$ \\
Responsável pela internação & & \\
Pais & 140 & 40,4 \\
Cônjuge & 76 & 21,9 \\
Irmão(a) & 70 & 20,2 \\
Outros & 43 & 12,3 \\
Filhos & 18 & 5,2 \\
Total & 347 & $100 \%$ \\
Não informado & 3 & \\
Encaminhamento para internação & & \\
Sim & 347 & 99,1 \\
Não & 3 & 0,9 \\
Total & 350 & $100 \%$ \\
\hline
\end{tabular}

(n-129) 36,9\%. A internação voluntária é predominante em (n-340) 97,1\%. Entre os responsáveis legais pela internação, houve o predomínio dos pais em (n-140) $40,4 \%$. Dentre os pacientes assistidos na unidade (n347 ), 99,1\% foram encaminhados para hospitalização a partir de outros serviços.

A tabela 2 mostra os diagnósticos de transtornos mentais e comportamentais devidos ao uso de substâncias psicoativas levantados conforme a Classificação Internacionais de Doenças (CID-10). O diagnóstico com maior predominância foi o Transtornos mentais e comportamentais devido ao uso de múltiplas drogas (CID F-19), em (n:173) 49,4\%. Dentre os diagnósticos (n-347) 99,4\%, relacionava-se à Síndrome de Abstinência.

Nas características específicas da internação, a tabela 3 aponta que o período total de permanência dos dependentes químicos na instituição preponderou em entre a faixa de 31 a 40 dias. Quanto ao tipo de alta, a mais comum foi a alta clínica. No entanto, o abandono ao tratamento é quantitativamente relevante, visto que (n-145) 41,4\% dos pacientes não aderiram ao tratamento completo, seja por indisciplina, evasão ou alta a pedido. Destaca-se que houve o predomínio de dependentes químicos que obtiveram encaminhamento para continuidade do tratamento em serviço extra-hospitalares.

$\mathrm{Na}$ tabela 4, a relação entre o tipo de droga consumida e o tipo de alta aponta que os dependentes de álcool aderem mais ao tratamento em comparação com os dependentes de crack, pois (n-135) 70,31\% dos alcoolistas tiveram alta clínica e para os compulsivos pelo crack ocorreu apenas com (n-59) 38,56\%. Nota-se que os usuários de crack aderiram menos ao tratamento (n-89) 58,16\%, em decorrência de alta a pedido, indisciplinar e por evasão, quando comparado a outras substâncias psicoativas.

Com relação ao histórico de tratamentos anteriores, os dados apontam que, dentre os 350 dependentes químicos, (n-251) 71,7\% foram internados anteriormente para tratamento da dependência química. O número de internações anteriores variou entre 1 (n-77) $33,48 \%$ e 30 (n-1) $0,48 \%$, sendo que a faixa de 1 a 5 internações predominou em $89,1 \%$.

No que se refere à variável de tratamentos extrahospitalares (n-348), evidencia-se que (n-244) 70,1\% dos pacientes nunca realizaram algum tratamento extra-hospitalar para dependência química. Dentre os (n-104) $29,9 \%$ que realizaram algum tratamento extra-hospitalar, o CAPSad foi o mais frequentado, em $73 \%$ dos casos. 
Tabela 2 - Distribuição de diagnósticos psiquiátricos entre dependentes químicos. Curitiba, 2011

\begin{tabular}{lcc}
\hline Diagnósticos psiquiátricos & N & $\mathbf{\%}$ \\
\hline $\begin{array}{l}\text { Diagnósticos psiquiátricos (CID-10) } \\
\text { F-19 Transtornos mentais e com- }\end{array}$ & \\
portamentais devidos ao uso de & 173 & 49,4 \\
múltiplas drogas & & \\
$\begin{array}{l}\text { F-10 Transtornos mentais e com- } \\
\text { portamentais devidos ao uso de }\end{array}$ & 150 & 42,9 \\
álcool & & \\
F-14 Transtornos mentais e com- & & \\
portamentais devidos ao uso de & 27 & 7,7 \\
cocaína & & \\
Total & 350 & $100 \%$ \\
Relacionado a: & & \\
Síndrome de dependência & 347 & 99,4 \\
Intoxicação aguda & 1 & 0,3 \\
Síndrome psicótica & 1 & 0,3 \\
Total & 349 & $100 \%$ \\
Não informado & 1 & \\
\hline
\end{tabular}

Tabela 3 - Distribuição dos dados da alta hospitalar entre dependentes químicos. Curitiba, 2011

\begin{tabular}{lcc}
\hline Dados da alta hospitalar & N & \% \\
\hline Tempo de permanência & & \\
Até 10 dias & 77 & 22 \\
11 a 20 dias & 43 & 12,3 \\
21 a 30 dias & 42 & 12 \\
31 a 40 dias & 117 & 33,4 \\
Acima de 40 dias & 71 & 20,3 \\
Total & 350 & $100 \%$ \\
Tipo de alta & & \\
Clínica & 194 & 55,4 \\
Pedido & 103 & 29,4 \\
Indisciplinar & 22 & 6,3 \\
Evasão & 20 & 5,7 \\
Transferência & 11 & 3,2 \\
Total & 350 & $100 \%$ \\
Encaminhamento & & \\
Sim & 301 & 86 \\
Não & 49 & 14 \\
Total & 350 & $100 \%$ \\
\hline
\end{tabular}

Tabela 4 - Relação entre o tipo de droga consumida e o tipo de alta. Curitiba, 2011

\begin{tabular}{lcccc}
\hline $\begin{array}{l}\text { Tipo de alta (N) } \\
\text { (\%) }\end{array}$ & Álcool & Crack & Cocaína & Maconha \\
\hline Pedido & 44 & 56 & 1 & 2 \\
& $22,92 \%$ & $36,60 \%$ & $100 \%$ & $50 \%$ \\
Clínica & 135 & 59 & - & - \\
& $70,31 \%$ & $38,56 \%$ & - & - \\
Indisciplinar & 4 & 18 & - & - \\
& $2,08 \%$ & $11,76 \%$ & - & $0 \%$ \\
Evasão & 4 & 15 & - & 1 \\
& $2,08 \%$ & $9,80 \%$ & - & $25 \%$ \\
Transferência & 5 & 5 & - & 1 \\
& $2,61 \%$ & $3,28 \%$ & - & $25 \%$ \\
Total & 192 & 153 & 1 & 4 \\
\hline
\end{tabular}

\section{DISCUSSÃO}

Os transtornos ocasionados pelo uso abusivo de substâncias psicoativas causam notáveis impactos para o usuário, para sua família e para a sociedade, tais como perdas econômicas, danos à saúde, comprometimento das relações sociais e até mesmo perda de vínculos familiares ${ }^{(4)}$. Os dados desta pesquisa referentes ao estado civil confirmam que o uso de substância compromete as relações e os vínculos familiares e de relacionamento. Neste estudo, a média de idade identifica-se como produtiva quando é relacionada à vida laboral. No entanto, houve o predomínio de sujeitos desempregados no momento da internação. Esses dados corroboram aos achados da literatura ${ }^{(10)}$ a qual afirma que o dependente químico comumente é afastado das suas funções sociais e, consequentemente, do seu emprego, pois a ação de consumir a droga ocupa função central, em detrimento de outras atividades.

A permissividade da sociedade com relação às substâncias lícitas, em conjunto com a falta de cumprimento de leis em relação ao uso inadequado 
de drogas contribui para a exposição precoce, ainda na adolescência, período em que o indivíduo está em desenvolvimento e apresenta-se vulnerável ${ }^{(11)}$. E como resultado, as drogas ocasionam prejuízos de percepções, memória e pensamentos, resultando em deficit de aprendizagem, de rendimento e consequente abandono escolar ${ }^{(12)}$. Este fato foi evidenciado nesta pesquisa, pois há predomínio de pacientes com ensino restrito ao nível fundamental.

Quanto ao motivo de internação, observa-se que preponderou o uso crônico do álcool. Esse dado vai ao encontro da situação nacional, em que há mais dependentes de álcool que de outras substâncias psicoativas $^{(13)}$. O consumo de álcool é uma prática difundida entre diversos povos, com início cada vez mais precoce e em quantidade exacerbada, devido, entre outras causas, a sua legalidade. Trata-se de uma droga de baixo custo, acessível, presente nas ocasiões sociais, como reuniões com amigos ou com familiares ${ }^{(14)}$. Além disso, a mídia influencia tal prática, por meio de propagandas que estimulam o seu consumo, mostrando-o como ato meramente prazeroso ${ }^{(11)}$.

Nos últimos anos os atendimentos em saúde mental deslocaram-se do regime hospitalocêntrico para os atendimentos ambulatoriais e extra-hospitalares, porém há situações específicas em que a hospitalização se faz necessária. Nesse sentido, a legislação especifica que a internação hospitalar deve ser autorizada pelo paciente, por meio de um consentimento livre e esclarecido, quando este for capaz de julgar e decidir sobre tal procedimento $^{(15)}$. A perspectiva de legalidade se reflete nesta pesquisa, em que a maioria dos dependentes químicos internou de forma voluntária.

No que se refere aos responsáveis legais pela internação, os dados apresentam a preponderância dos familiares, principalmente dos pais. Essa estatística corrobora com um estudo realizado com dependentes químicos, no qual $66,9 \%$ dos usuários de álcool estavam acompanhados por pais na internação ${ }^{(12)}$. A família representa papel fundamental na formação de seus membros, e a integração destes no tratamento mostra-se uma das principais ferramentas de recuperação e reinserção social. Com o apoio familiar, o dependente mantém-se motivado para iniciar e manter o tratamento, bem como prevenir a recaída ${ }^{(16)}$.

O número elevado de encaminhamentos para a internação, a partir de outra instituição, configura as redes entre serviços de atenção aos usuários de drogas, preconizada a partir das diretrizes do Ministério da Saúde, que normatizam a atenção integral ao usu- ário de álcool e drogas a partir da intersetorialidade e intrasetorialidade ${ }^{(4)}$.

Quanto ao uso de duas ou mais substâncias psicoativas justifica-se pela prevalência do diagnóstico de Transtornos mentais e comportamentais devidos ao uso de múltiplas drogas (F-19) de acordo com a CID-10. Cabe salientar que, ao diagnosticar o F-19 nos registros dos prontuários, os profissionais não consideraram $\mathrm{o}$ tabaco como policonsumo. A prevalência deste diagnóstico mostra-se discrepante em relação ao motivo de internação, pois, na admissão no hospital, o dependente químico, na maioria das vezes, relata apenas a droga de preferência como motivo para se internar. Uma pesquisa com dependentes químicos hospitalizados realizados em Curitiba, Paraná, apontou também o diagnóstico F-19 como o mais preponderante na população em estudo, com $53,6 \%{ }^{(12)}$. O policonsumo mostra-se cada vez mais evidente, pois os dependentes de apenas uma substância psicoativa tornam-se dependentes de outras a fim de atenuar, intensificar ou modificar a característica de sua intoxicação, ou até mesmo com o intuito de aliviar os sintomas da síndrome de abstinência ${ }^{(10)}$.

Os diagnósticos listados nesta pesquisa estão relacionados, em sua maioria, à Síndrome de Dependência. Por conseguinte, há prevalência de sujeitos que apresentam um agrupamento de sintomas comportamentais, cognitivos e fisiológicos resultante do consumo de substâncias psicoativas. Portanto, não possuem mais controle sobre o consumo dessa substância, que se torna a unidade funcional das suas vidas. Por meio do prazer de um simples ato, o consumo das drogas torna-se essencial para o funcionamento físico e psíquico ${ }^{(10)}$.

Quanto ao período das internações, a maior prevalência foi na faixa de 31 a 40 dias. Este achado exprime a necessidade de o serviço manter o paciente internado o mais breve possível, como preconiza a Lei Federal 10.216/2001 da Reforma Psiquiátrica. A partir da vigência desta lei, o atendimento aos portadores de dependência química ou de outros transtornos mentais passa a ser redirecionado com o enfoque da desinstitucionalização e com o propósito de reabilitação psicossocial com tratamentos extra-hospitalares ${ }^{(15)}$.

A elevada taxa de abandono ao tratamento hospitalar por alta a pedido, indisciplina ou evasão, possivelmente, relaciona-se com a concepção que os dependentes químicos possuem de não haver problemas relacionados ao consumo de drogas. Esses dados corroboram com uma pesquisa realizada em Porto Alegre, Rio Grande do Sul, em que 69,3\% dos depen- 
dentes químicos que não aderiram ao tratamento não possuíam motivação para mudança de comportamento adicto e para o tratamento ${ }^{(7)}$.

Além dos fatores motivacionais, este estudo apontou que o dependente de crack tem menor adesão ao tratamento quando se relaciona a outras substâncias psicoativas. Corroborando com esta pesquisa um estudo realizado com dependentes de cocaína, na forma de crack e/ou em pó, destacou que 208 usuários $(47,3 \%)$ registravam várias internações, mas não concluíram o tratamento hospitalar por abandono, fuga ou a pedido(8).

O uso do crack caracteriza-se por um alto potencial aditivo devido à intensa fissura, por conseguinte, estimula o consumo repetitivo e exacerbado. Deste modo, a compulsão incita o dependente de crack a buscar obcecadamente a substância por meio de comportamentos de riscos, que procedem em impactos sociais e pessoais ${ }^{(17)}$. Nesse sentido, é inegável reconhecer que os índices de criminalidade e violência por dependentes químicos, estão relacionados ao consumo de crack e suas consequências ${ }^{(8,17)}$. Diante deste contexto, os dependentes de crack apresentam menor adesão ao tratamento. Considerando esta realidade, a assistência aos dependentes de crack deve abordar outras formas além dos modelos de tratamento convencionais. Para isto, é necessário o uso de abordagens mais intensivas, que considerem os aspectos comumente referentes a essa clientela, tais como a fissura intensa e suas consequências ${ }^{(17)}$.

As internações hospitalares para dependência química não devem ser prolongadas a fim de que o paciente não perca os vínculos sociais. Com isso, fazse necessária, após a alta, a adoção dos tratamentos que auxiliam na manutenção da abstinência, por meio de estratégias como a farmacoterapia, psicoterapia e reabilitação social ${ }^{(18)}$. Nesta perspectiva, os dependentes químicos desta pesquisa, após receber alta, foram encaminhados e/ou orientados para sequenciar o tratamento extra-hospitalar e em participação de grupos de ajuda mútua.

A existência dos grupos de ajuda mútua, datam das primeiras décadas do século XX. Não são serviços de saúde, mas integram uma rede de apoio e auxiliam o usuário de drogas na reinserção social, na promoção de vínculos benéficos e na manutenção da abstinência, pois, ao se envolver com a recuperação de outros dependentes, há a reafirmação da esperança na sua própria recuperação ${ }^{(19)}$.
Outro exemplo de serviço substitutivo é o Centro de Atenção Psicossocial Álcool e Drogas (CAPSad). Este serviço possui uma equipe multidisciplinar, com o propósito de recuperação dos dependentes quími$\cos$, enfatizando a reabilitação e reinserção social do usuário, reduzindo fatores de risco para o consumo e minimizando o preconceito e estigma social frente à problemática da dependência química ${ }^{(4)}$.

A dependência química é um transtorno crônico e de difícil recuperação, devido à sua complexidade e aos elevados custos econômicos envolvidos. Porém, a maior limitação da recuperação é a manutenção da abstinência, proveniente da própria natureza deste transtorno, que apresenta propensão à recaída ${ }^{(9,14)}$. Ao sair do meio hospitalar, o dependente, na maioria das vezes, tem dificuldade em tolerar os problemas e, como consequência, retoma o uso da substância, o que leva à recaída ${ }^{(14)}$. A reincidência do consumo de drogas faz com que o dependente químico procure novamente o tratamento e seja reinternado. Nesse sentido, a participação de pessoas com transtorno mentais em serviços extra-hospitalares, entre elas os dependentes químicos, mostra-se essencial para a redução do número de hospitalizações, pois ajuda a romper o ciclo de reinternações ${ }^{(5)}$. Ao participar destes serviços, o indivíduo pode conciliar o tratamento com sua vida social sem precisar se afastar integralmente de determinadas ocupações cotidianas.

Reafirmando esta perspectiva, os resultados evidenciam o elevado número de indivíduos que internaram ao menos uma vez para tratamento da dependência, o que possivelmente se relaciona à baixa procura aos tratamentos extra-hospitalares e grupos de ajuda mútua, pois apenas $29,9 \%$ dos pacientes participaram em algum momento destes locais.

Apesar da implementação de uma política específica à atenção aos usuários de drogas que prioriza os serviços extra-hospitalares, percebe-se a baixa adesão aos tratamentos por desassistência ao usuário, formas de tratamento ainda deficientes, estigmas e preconceitos e relação do indivíduo com a criminalidade ${ }^{(20)}$.

Para assegurar o fim do fenômeno de reinternações subsequentes, um Hospital Psiquiátrico do Rio Grande do Sul desenvolveu um projeto denominado Alta Assistida, com o qual se objetiva manter o tratamento dos portadores de transtornos mentais no âmbito comunitário. Para a efetividade desse projeto, a equipe multidisciplinar entra em contato com pacientes e familiares após a alta para avaliar a recuperação, orientar e direcionar o tratamento ${ }^{(5)}$. Diante 
do exposto, a presença de uma alta acompanhada na perspectiva da dependência química aponta para uma ferramenta possivelmente eficaz para a redução do número de internações.

\section{CONSIDERAÇÕES FINAIS}

A partir dos resultados obtidos, conclui-se que a adesão ao tratamento ainda é um desafio, devido à alta taxa de abandono hospitalar, principalmente no que concerne aos dependentes de crack. Deste modo, evidencia-se a necessidade de implementar um plano de cuidados específicos para dependentes de crack, que considere as particularidades do mecanismo de ação desta droga e de suas consequências.

$\mathrm{O}$ alto número de internações anteriores para dependência química indica a dificuldade que os dependentes químicos possuem em manterem-se abstinentes após a internação. Tal fato está relacionado à baixa adesão dos pacientes aos serviços extra-hospitalares. Por conseguinte, a falta de acompanhamento após a internação favorece a recaída. Com isso, salienta-se a necessidade de os órgãos governamentais instituírem mais serviços extra-hospitalares que atendam a demanda populacional, fornecendo uma assistência de qualidade, para que haja o aumento de adesão dos dependentes químicos a eles.

Esta pesquisa apresenta limitações no que diz respeito apenas à coleta de dados de prontuários, devido a falta de informações, falhas de preenchimento, ilegibilidade de alguns registros e até mesmo incongruência de dados. Portanto, nota-se a necessidade do preenchimento adequado deste documento por todos os profissionais que prestem atendimento ao paciente, pois se trata de um documento de respaldo legal, no qual há registro de todos os cuidados e intervenções prestadas.

Contudo, todas as variáveis abordadas possibilitam a compreensão do processo de internação de dependentes químicos em uma unidade de reabilitação, o que subsidia a construção de estratégias de assistência embasadas na realidade investigada, concomitante às políticas públicas de atenção a usuários de álcool e outras drogas e à política de saúde mental do Brasil.

Considerando a existência de poucos estudos sobre esta temática, espera-se que as questões levantadas nesta pesquisa sejam aprofundadas em novas investigações, que poderão contribuir para alimentar o debate sobre o tema, uma vez que a dependência química configura uma doença crônica e de difícil recuperação.

\section{REFERÊNCIAS}

1. World Health Organization. ATLAS on substance use: Resources for the prevention and treatment of substance use disorder. Geneva: World Health Organization; 2010.

2. UNODC - United Nations Office on Drugs and Crime. World drug report. New York: United Nations Office on Drugs and Crime; 2011.

3. World Health Organization. Substance Abuse. Geneva: World Health Organization; 2011. [Acesso em 23 jul 2011]. Disponível: http://www.who.int/topics/ substance abuse/en/

4. Ministério da Saúde (BR). A política do Ministério da Saúde para a atenção integral a usuários de álcool e outras drogas. $2^{\mathrm{a}}$ ed. Brasília: Ministério da Saúde; 2004.

5. Bezerra CG, Dimenstein M. O fenômeno da reinternação: um desafio à Reforma Psiquiátrica. Mental. 2011;9(16): 417-41.

6. Ferreira FGKY, Luz JA, Obrzut Neto L, Santos KA. Uma visão multiprofissional humanizada no tratamento da pessoa com dependência química em enfermaria psiquiátrica de um hospital geral no Paraná. Cogitare enferm. 2005;10(2):54-62.

7. Oliveira MS, Szupszynski KDR, DiClemente C. Estudo dos estágios motivacionais no tratamento de adolescentes usuários de substâncias psicoativas ilícitas. Psico. 2010;41(1):40-6.

8. Ferreira Filho OF, Turchi MD, Laranjeira R, Castelo A. Perfil sociodemográfico e de padrões de uso entre dependentes de cocaína hospitalizados. Rev. saude publica. 2003;37(6):751-9.

9. Santos CE, Costa-Rosa A. A experiência da toxicomania e da reincidência a partir da fala dos toxicômanos. Estud. psicol. (Campinas) 2007;24(4):487-502.

10. Ribeiro PL, Andrade, AG. Transtornos mentais relacionados ao uso de substâncias psicoativas. In: Louzã Neto MR, Elkis H. Psiquiatria básica. $2^{\mathrm{a}}$ ed. Porto Alegre: Artmed; 2007. p. 195-210

11. Pechansky F, Szobot CM, Scivoletto S. Uso de álcool entre adolescentes: conceitos, características epidemiológicas e fatores etiopatogênicos. Rev. bras. psiquiatr. 2004; 6 Supl 1:14-7.

12. Alves R, Kossobudzky LA. Caracterização dos adolescentes internados por álcool e outras drogas na

Cogitare Enferm. 2012 Jul/Set; 17(3):444-51 
cidade de Curitiba. Interação Psicol. 2002;6(1):65-79.

13. Carlini EA, Gladuróz JC. II Levantamento Domiciliar sobre o Uso de Drogas Psicotrópicas no Brasil: estudo envolvendo 108 maiores cidades do país. São Paulo: CEBRID/UNIFESP; 2005.

14. Büchelle F, Marcatti M, Rabelo DR. Dependência química e prevenção à recaída. Texto Contexto Enferm. 2004;13(2):233-40.

15. Brasil. Lei n. 10.216, de 6 de abril de 2001. Dispõe sobre a proteção e os direitos das pessoas portadoras de transtornos mentais e redireciona o modelo assistencial em saúde mental. Diário Oficial da República Federativa do Brasil, Brasília, 6 abr, 2001.

16. Silva LHP, Borba LO, Paes MR, Guimarães NA, Mantovani MF, Maftum MA. Perfil dos dependentes químicos atendidos em uma unidade de reabilitação de um hospital psiquiátrico. Esc. Anna Nery. 2010;14(3):585-90.

17. Chaves TV, Sanchez ZM, Ribeiro LA, NAPPO AS. Fissura por crack: comportamentos e estratégias de controle de usuários e ex-usuário. Rev. Saúde Pública. 2011;45(6):1168-75.

18. Sousa FSP, Oliveira EM. Caracterização das internações de dependentes químicos em Unidade de Internação Psiquiátrica do Hospital Geral. Cienc saude colet. 2010;15(3):671-7.

19. Rigotto SD, Gomes WB. Contextos de abstinência e de recaída na recuperação da dependência química. Psicol: teor. e pesq. 2002;18(1):95-106.

20. Azevedo DM, Miranda FAN, Scivoletto S. Práticas profissionais e tratamentos ofertados nos CAPSad do município de Natal - RN: com a palavra família. Esc. Anna Nery. 2010;14(1):56-63. 